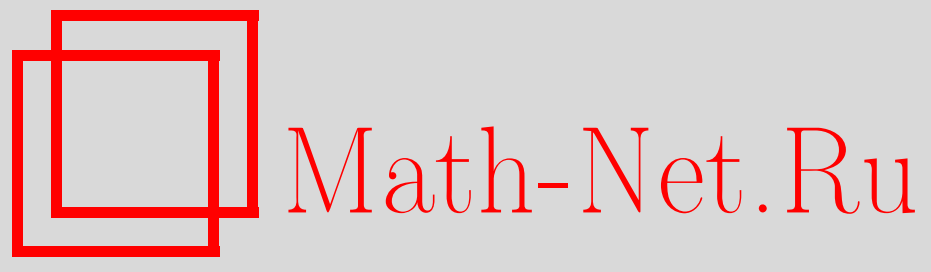

Я. Г. Синай, Предельная теорема для тригонометрических сумм. Теория завитков, УМН, 2008, том 63, выпуск $6,31-38$

DOI: https://doi.org/10.4213/rm9250

Использование Общероссийского математического портала Math-Net.Ru подразумевает, что вы прочитали и согласны с пользовательским соглашением http://www . mathnet.ru/rus/agreement

Параметры загрузки:

IP : 54.80 .97 .219

26 апреля 2023 г., $11: 28: 11$

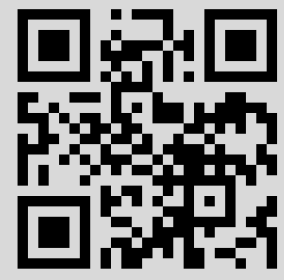




\title{
Предельная теорема для тригонометрических сумм. Теория завитков
}

\author{
Я. Г. Синай \\ В настоящей статье мы обсуждаем поведение тригонометрических сумм \\ $\sum \exp \left\{2 \pi \alpha n^{2}\right\}$ и их предельного распределения как функции от $N$. Ана- \\ лиз основан на применении метода ренормгруппы. \\ Библиография: 19 названий.
}

Текст этот заметки в значительной степени воспроизводит мой доклад на конференции, посвященной 70-летию С. П. Новикова. Я желаю Сергею Петровичу крепкого здоровья, энергии и новых замечательных результатов.

Новиков учился в группе, в которой лет 50 тому назад я преподавал теорию вероятностей. Тогда он был поглощен актуальными топологическими задачами и мы практически не встречались. Мне хотелось бы использовать возможность, предоставленную настоящей конференцией, и продемонстрировать Сергею Петровичу, что в теории вероятностей тоже есть красивые задачи и глубокие содержательные результаты.

Я признателен аспиранту Принстонского университета Ф. Челларози за многочисленные обсуждения и за помощь в подготовке текста к публикации.

Для фиксированных $a \in(-1,1] \backslash\{0\}$ и $N \in \mathbb{N}$ рассмотрим тригонометрическую сумму

$$
\mathscr{S}_{a}(N)=\sum_{n=0}^{N-1} \exp \left\{i \pi a n^{2}\right\} .
$$

Тогда

$$
\mathscr{S}_{-a}(N)=\overline{\mathscr{S}_{a}(N)} \quad \text { и } \quad \int_{-1}^{1}\left|\mathscr{S}_{a}(N)\right|^{2} \mathrm{~d} a=N .
$$

Мы изучим поведение $\mathscr{S}_{a}(N)$ как функции $N$ для типичных значений $a$. Этот вопрос обсуждался во многих работах, в частности, Харди и Литтлвудом [1], Г. Вейлем [2], ван дер Корпутом [3], Морделлом [4], Уильтоном [5] и многими другими. Среди относительно недавних работ можно упомянуть Куциаса и Казариноффа [6], [7], Берри и Гольдберга [8], Марклофа [9], [10], а также Федотова и Клоппа [11]. Работа [8] была, по-видимому, первой, в которой подробно исследовалась зависимость от $N$. Тот, кто знаком с теорией вероятностей, мог бы ожидать появления кривых, напоминающих двумерное броуновское

(C) Я.Г. СинАй, 2008 

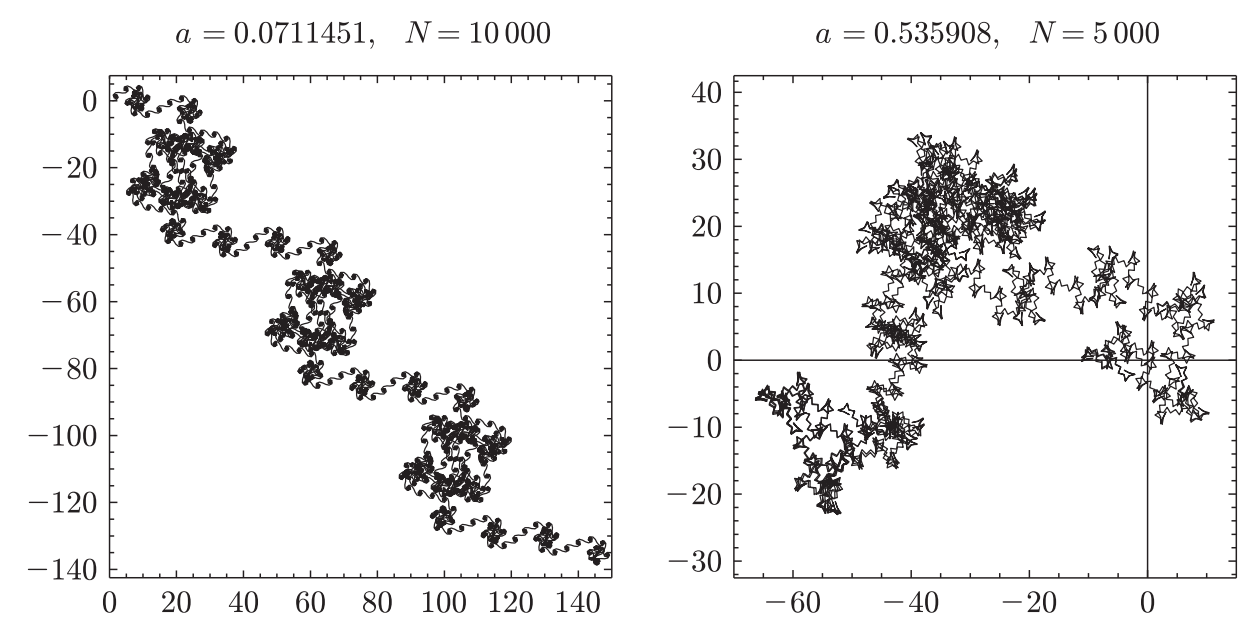

Рис. 1. Две кривые, порожденные тригонометрическими суммами

движение - самый устойчивый и широко распространенный тип предельных случайных кривых. Однако численный анализ показывает, что наблюдаемые кривые имеют очевидную масштабно-инвариантную структуру, мало похожую на броуновское движение. Рис. 1 дает представление о виде $\left\{\mathscr{S}_{a}(n)\right\}_{n=1}^{N}$ для $a=0.0711451$ и $N=10000$ (слева), а также для $a=0.535908$ и $N=5000$ (справа). Мы видим, что $\left\{\mathscr{S}_{a}(n)\right\}_{n=1}^{N}$ состоит из спиралевидных компонент разного масштаба, соединенных регулярными участками. Такие спирали называются завитками, и задача теории состоит в обсуждении их вида и свойств.

Возьмем точки $\mathscr{S}_{a}(n-1), \mathscr{S}_{a}(n), \mathscr{S}_{a}(n+1)$ и обозначим через $\rho_{a}(n)$ радиус окружности, проходящей через них. Назовем $\rho_{a}(n)$ локалъным дискретным радиусом кривизны. Легко проверяется, что

$$
\rho_{a}(n)=\frac{1}{2}\left|\operatorname{cosec} \frac{\pi a(2 n-1)}{2}\right| .
$$

Функция $f(t)=|\operatorname{cosec} t|$ периодическая с периодом $\pi$, следовательно,

$$
\rho_{a}(\nu)=\frac{1}{2}\left|\operatorname{cosec} \frac{\pi a(2 \nu-1)}{2}\right|
$$

является $1 / a$-периодической функцией. Ее локальные экстремумы расположены в точках $(2 k+1) /(2 a)+1 / 2$, и она имеет вертикальные асимптоты при $k / a+1 / 2, k \in \mathbb{Z}$. Локальный дискретный радиус кривизны получается как значения $\rho_{a}$ в целых числах.

Для малых $a$ на каждом периоде лежит порядка $1 /|a|$ целых точек и структуру с завитками легко объяснить: тем $n$, для которых $\rho_{a}(n)$ велико, соответствуют почти прямые участки $\left\{\mathscr{S}_{a}(n)\right\}_{n=1}^{N}$, а точки, близкие к минимумам $\rho_{a}$, дают спиралевидные участки. При $a \sim 1$ завитки пропадают. Однако мы продемонстрируем, как такие завитки возникают в других масштабах. 
Главный шаг всей теории основан на приближенном "ренормгрупповом" уравнении

$$
\mathscr{S}_{a}(N) \sim \exp \left\{\frac{\pi}{4} i\right\}|a|^{-1 / 2} \mathscr{S}_{a_{1}}\left(N_{1}\right),
$$

где $a_{1} \equiv-1 / a(\bmod 2)$ и $N_{1}=\lfloor|a| N\rfloor$. Точнее,

$$
\left.\left.\left|\mathscr{S}_{a}(N)-\exp \left\{\frac{\pi}{4} i\right\}\right| a\right|^{-1 / 2} \mathscr{S}_{a_{1}}\left(N_{1}\right)\left|\leqslant C_{1}\right| a\right|^{-1 / 2}+C_{2}
$$

с абсолютными константами $C_{1}, C_{2}$, не зависящими от $N$. Вывод оценки (1) опирается на формулу суммирования Пуассона

$$
\sum_{-\infty<n<\infty} g(n)=\sum_{-\infty<m<\infty} \hat{g}(m)
$$

где

$$
\hat{g}(m)=\int_{-\infty}^{\infty} \exp \{-2 \pi i m x\} g(x) \mathrm{d} x .
$$

В нашем случае эта формула дает

$$
\begin{aligned}
\mathscr{S}_{a}(N) & =\sum_{n=0}^{N-1} \exp \left\{\pi i a n^{2}\right\}=\sum_{-\infty<n<\infty} \exp \left\{\pi i a n^{2}\right\} \chi_{[0, N-1]}(n) \\
& =\sum_{-\infty<m<\infty} \int_{-\infty}^{\infty} \exp \{-2 \pi i m x\} \exp \left\{\pi i a x^{2}\right\} \chi_{[0, N-1]}(x) \mathrm{d} x \\
& =\sum_{-\infty<m<\infty} \int_{0}^{N-1} \exp \left\{\pi i\left[a x^{2}-2 m x\right]\right\} \mathrm{d} x .
\end{aligned}
$$

Для оценки правой части (2) мы используем асимптотический метод Лапласа (метод стационарной фазы). Основной вклад в (2) вносят интегралы по малым окрестностям критических точек

$$
\frac{\mathrm{d}}{\mathrm{d} x}\left(a x^{2}-2 m x\right)=0, \quad \text { т. е. } x_{m}=\frac{m}{a} .
$$

Легко показать, что достаточно рассмотреть критические точки с $0 \leqslant m<$ $\lfloor|a| N\rfloor$ : вклад остальных точек равномерно ограничен. Мы оставляем подробности читателю.

Мы используем отображение

$$
U:(-1,1] \backslash\{0\} \rightarrow(-1,1] \backslash\{0\}, \quad \text { где } U(t)=-\frac{1}{t}(\bmod 2) .
$$

График $U$ приведен на рис. 2 ; он состоит из счетного числа гладких компонент. Каждый открыто-замкнутый интервал $O_{k}=(1 /(2 k+1), 1 /(2 k-1)]$ взаимно однозначно отображается на $(-1,1]$ по формуле $t \mapsto-1 / t+2 k$. Пусть

$$
B(k,-1)=\left(\frac{1}{2 k}, \frac{1}{2 k-1}\right], \quad B(k,+1)=\left(\frac{1}{2 k+1}, \frac{1}{2 k}\right], \quad k \in \mathbb{N} .
$$




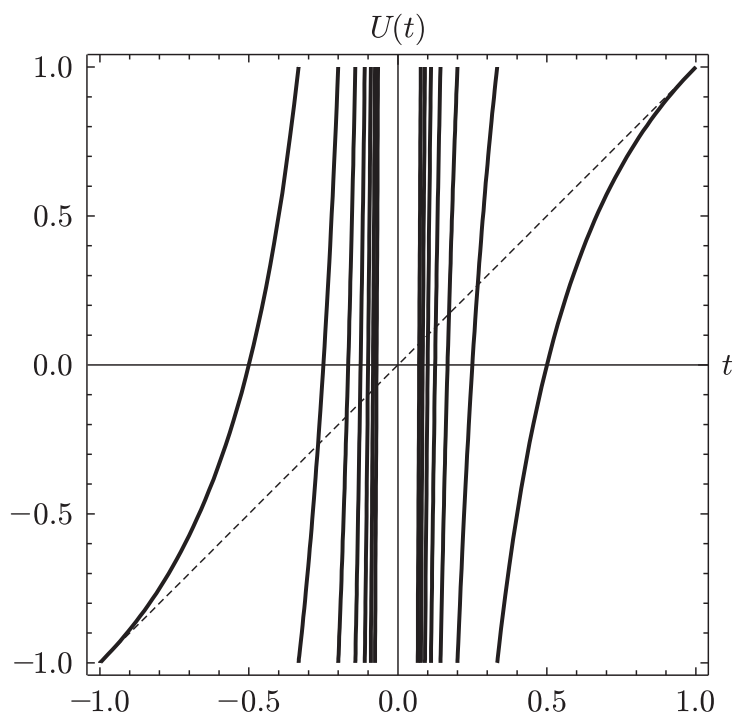

Рис. 2. График отображения $U$

Для $\alpha=\alpha_{0}$ из $B(k, \xi), \alpha_{j}=T^{j} \alpha_{0}, \eta_{j+1}=\eta\left(\alpha_{j}\right)$ мы имеем $N_{j+1}=\left\lfloor\alpha_{j} N_{j}\right\rfloor$ и ренормгрупповую формулу

$$
\mathscr{S}_{\alpha}^{(\eta)}(N)= \begin{cases}\mathscr{S}_{\alpha}(N) & \text { при } \eta=+1 \\ \overline{\mathscr{S}_{\alpha}(N)} & \text { при } \eta=-1\end{cases}
$$

Нам потребуется также отображение $T$, задаваемое формулой

$$
T \alpha=\xi \cdot\left(\frac{1}{\alpha}-2 k\right) \quad \text { при } \alpha \in B(k, \xi), k \in \mathbb{N}, \xi \in\{ \pm 1\} \text {. }
$$

Теперь мы можем записать ренормгрупповую формулу (1) следующим образом:

$$
\mathscr{S}_{\alpha_{0}}(N)=\exp \left\{\frac{\pi}{4} i\right\} \alpha_{0}^{-1 / 2} \mathscr{S}_{\alpha_{1}}^{\left(\eta_{1}\right)}\left(N_{1}\right)+\Lambda\left(\alpha_{0}, N\right)
$$

где $\alpha_{1}=T \alpha_{0}, \eta_{1}=\eta\left(\alpha_{0}\right)$ и $\left|\Lambda\left(\alpha_{0}, N\right)\right| \leqslant C_{1} \alpha_{0}^{-1 / 2}+C_{2}$. Полагая

$$
\kappa(\alpha, s)=1+\eta_{1}+\eta_{1} \eta_{2}+\cdots+\eta_{1} \eta_{2} \cdots \eta_{s-1}
$$

и

$$
\widetilde{\Lambda}\left(\alpha_{j}, N_{j}\right)=\exp \left\{\kappa(\alpha, j) \frac{\pi}{4} i\right\} \cdot \Lambda^{\left(\eta_{1} \eta_{2} \cdots \eta_{j}\right)}\left(\alpha_{j}, N_{j}\right)
$$


получаем

$$
\begin{aligned}
\mathscr{S}_{\alpha_{0}}(N)=\widetilde{\Lambda} & \left(\alpha_{0}, N\right)+\alpha_{0}^{-1 / 2} \widetilde{\Lambda}\left(\alpha_{1}, N_{1}\right)+\left(\alpha_{0} \alpha_{1}\right)^{-1 / 2} \widetilde{\Lambda}\left(\alpha_{2}, N_{2}\right)+\cdots \\
& +\left(\alpha_{0} \cdots \alpha_{j-1}\right)^{-1 / 2} \widetilde{\Lambda}\left(\alpha_{j}, N_{j}\right)+\cdots \\
& +\left(\alpha_{0} \cdots \alpha_{r-2}\right)^{-1 / 2} \widetilde{\Lambda}\left(\alpha_{r-1}, N_{r-1}\right) \\
& +\exp \left\{\kappa(\alpha, r) \frac{\pi}{4} i\right\}\left(\alpha_{0} \cdots \alpha_{r-1}\right)^{-1 / 2} \mathscr{S}_{\alpha_{r}}^{\left(\eta_{1} \cdots \eta_{r}\right)}\left(N_{r}\right) .
\end{aligned}
$$

Теперь возникает важный вопрос о выборе $r$. Это имеет отношение к так называемым задачам типа восстановления в эргодической теории. В теории вероятностей задачи типа восстановления возникают следующим образом. Пусть $\gamma_{1}, \gamma_{2}, \ldots, \gamma_{n}, \ldots-$ последовательность таких независимых одинаково распределенных случайных величин, что $\gamma_{j} \geqslant 0, \mathrm{E} \gamma_{j}<\infty$. Выберем параметр $R$ (который будет впоследствии стремиться к бесконечности) и рассмотрим случайную величину $n$, равную первому моменту, когда выполняется неравенство

$$
\gamma_{1}+\cdots+\gamma_{n}>R
$$

Основной результат теории восстановления говорит, что разность

$$
\gamma_{1}+\cdots+\gamma_{n}-R
$$

имеет предельное распределение при $R \rightarrow \infty$. В некоторых случаях аналогичный результат выполняется, даже когда $\gamma_{j}$ имеют симметричное распределение и $\mathrm{E}\left|\gamma_{j}\right|=\infty$ (см. [12]). В совместной статье с К. Ульчиграй (см. [13]) мы доказали существование предельного распределения при $R \rightarrow \infty$ для отношения $q_{n}(\alpha) / R$, где $\alpha$ имеет найденное Гауссом распределение с плотностью

$$
p(x)=\frac{1}{(1+x) \log 2},
$$

a $q_{n}(\alpha)$ - первый превосходящий $R$ знаменатель подходящих дробей разложения $\alpha$ в непрерывную дробь. Этот результат также относится к теории восстановления.

Ф. Челларози [14] рассмотрел аналогичные вопросы для T. Ситуация здесь слегка отличается от обычных непрерывных дробей, потому что у $T$ есть только $\sigma$-конечная эргодическая инвариантная мера $\mu$ из-за наличия нейтральной неподвижной точки $\alpha=1$, и напоминает случай дробей Фарея и соответствующего отображения Фарея. (А именно, при $\alpha \in(1 /(k+1), 1 / k], k>1$, имеем $1 / \alpha=k+\theta, 0 \leqslant \theta<1$, и полагаем $F(\alpha)=(1 / \alpha-1)^{-1}$. При $k=1$ полагаем $F(\alpha)=1 / \alpha-1$.) Отображение Фарея также обладает только $\sigma$-конечной инвариантной мерой, и логарифмы знаменателей соответствующих дробей растут как $n / \log n$. Вернемся к отображению $T$. Его орбиты связаны с так называемыми четными непрерывными дробями (Швайгер [15], [16], Краайкамп и Лопес [17])

$$
\alpha=\frac{1}{2 k_{1}+\frac{\xi_{1}}{2 k_{2}+\frac{\xi_{2}}{2 k_{3}+\cdots}}}=\left[\left(k_{1}, \xi_{1}\right),\left(k_{2}, \xi_{2}\right), \ldots\right], \quad k_{j} \in \mathbb{N}, \quad \xi_{j} \in\{ \pm 1\} .
$$


Подходящие дроби для них имеют вид

$$
\frac{p_{n}}{q_{n}}=\frac{1}{2 k_{1}+\frac{\xi_{1}}{2 k_{2}+\frac{\xi_{2}}{2 k_{3}+\ddots}+\frac{\xi_{n-2}}{2 k_{n-1}+\frac{\xi_{n-1}}{2 k_{n}}}}} .
$$

Они удовлетворяют рекуррентным соотношениям

$$
\begin{aligned}
p_{n} & =2 k_{n} p_{n-1}+\xi_{n-1} p_{n-2}, \\
q_{n} & =2 k_{n} q_{n-1}+\xi_{n-1} q_{n-2},
\end{aligned}
$$

где $q_{-1}=p_{0}=0, p_{-1}=q_{0}=\xi_{0}=1$. Среди свойств этих непрерывных дробей отметим одно:

$$
\left(\alpha_{0} \cdots \alpha_{n-1}\right)^{-1}=q_{n}\left(1+\xi_{n} \alpha_{n} \frac{q_{n-1}}{q_{n}}\right) .
$$

Следующая теорема доказана $\Phi$. Челларози.

TeOPEма. Пусть $n_{R}=n_{R}(\alpha)=\min \left\{n \in \mathbb{N}: q_{n}>R\right\}$. Тогда отношение $q_{n_{R}} / R$ имеет предельное распределение при $R \rightarrow \infty$.

Вернемся к (3). Пусть $r$ - момент, для которого впервые выполняется

$$
\left(\alpha_{0} \cdots \alpha_{r-1}\right)^{-1 / 2}>\sqrt{N} .
$$

Тогда величины $N_{r}, \mathscr{S}_{\alpha_{r}}^{\left(\eta_{1} \cdots \eta_{r}\right)}\left(N_{r}\right)$ и $\left(\alpha_{0} \cdots \alpha_{r-1}\right)^{-1 / 2} N^{-1 / 2}$ являются $\mathscr{O}(1)$ и мы имеем

$$
\begin{aligned}
\frac{\mathscr{S}_{\alpha_{0}}(N)}{\sqrt{N}}=\exp \left\{\kappa(\alpha, r) \frac{\pi}{4} i\right\} \frac{\left(\alpha_{0} \cdots \alpha_{r-1}\right)^{-1 / 2}}{\sqrt{N}} \mathscr{S}_{\alpha_{r}}^{\left(\eta_{1} \cdots \eta_{r}\right)}\left(N_{r}\right) \\
+\frac{\left(\alpha_{0} \cdots \alpha_{r-2}\right)^{-1 / 2}}{\sqrt{N}} \widetilde{\Lambda}\left(\alpha_{r-1}, N_{r-1}\right)+\cdots \\
+\frac{\left(\alpha_{0} \cdots \alpha_{r-j}\right)^{-1 / 2}}{\sqrt{N}} \widetilde{\Lambda}\left(\alpha_{r-j+1}, N_{r-j+1}\right)+\cdots \\
+\frac{\alpha_{0}^{-1 / 2}}{\sqrt{N}} \widetilde{\Lambda}\left(\alpha_{1}, N_{1}\right)+\frac{1}{\sqrt{N}} \widetilde{\Lambda}\left(\alpha_{0}, N\right) .
\end{aligned}
$$

Произведение $\alpha_{r-j+1} \widetilde{\Lambda}\left(\alpha_{r-j+1}, N_{r-j+1}\right)$ также есть $\mathscr{O}(1)$ в силу $(1)$, а $j$-й член в (4) убывает как $\exp \{-(j / \log j)(1+\mathscr{O}(1))\}$ с ростом $j$. Эта скорость убывания вытекает из следующего результата, также недавно доказанного Ф. Челларози (этот результат является следствием теории, изложенной Ааронсоном в его книге по эргодической теории преобразований с бесконечной инвариантной мерой [18]). 
ТеОремА. Для всякой вероятностной меры $\mathrm{P}$ на $[0,1]$, являющейся абсолютно непрерывной относительно $\mu$, всякой $f \in L^{1}(\mu)$ и всякого $\varepsilon>0$

$$
\mathrm{P}\left(\left|\frac{\mathrm{S}_{n}(f)}{n / \log n}-\mu(f)\right| \geqslant \varepsilon\right) \longrightarrow 0 \quad \text { npu } n \rightarrow \infty,
$$

где $\mathrm{S}_{n}(f)$ - эргодическая сумма $\sum_{j=0}^{n-1} f \circ T^{j}$.

Аналогичный результат доказан в статье Кампанино и Изолы [19] для некоторого класса перемежающихся отображений с двумя ветвями.

Взяв $f(\alpha)=-\log \alpha$, мы получаем сходимость по вероятности

$$
-\frac{\log \left(\alpha_{0} \cdots \alpha_{n-1}\right)}{n / \log n} \longrightarrow \frac{\pi^{2}}{4} \quad \text { при } n \rightarrow \infty .
$$

Иными словами, в соответствующих эргодических теоремах имеет место сходимость по мере, а не с вероятностью 1, как в стандартном случае.

Формула (4) демонстрирует наличие завитков в различных масштабах и объясняет характер убывания этих масштабов.

\section{Список литературы}

[1] G.H. Hardy, J.E. Littlewood, "Some problems of diophantine approximation. II: The trigonometrical series associated with the elliptic $\vartheta$-functions", Acta Math., 37:1 (1914), 193-239.

[2] H. Weyl, "Über die Gleichverteilung von Zahlen mod. Eins", Math. Ann., 77:3 (1916), 313-352; рус. пер.: "О равномерном распределении чисел по модулю один", Герман Вейль. Избр. трудъ. Матем. Теорет. Физ., Классики науки, Наука, М., 1984, 58-93.

[3] J. G. van der Corput, "Über Summen, die mit den elliptischen $\vartheta$-Funktionen zusammenhängen", Math. Ann., 87:1-2 (1922), 66-77.

[4] L. J. Mordell, "The approximate functional formula for the theta function", J. London Math. Soc., 1 (1926), 68-72.

[5] J. R. Wilton, "The approximate functional formula for the theta function", J. London Math. Soc., 2 (1927), 177-180.

[6] E. A. Coutsias, N.D. Kazarinoff, "Disorder, renormalizability, theta functions and Cornu spirals", Phys. D, 26:1-3 (1987), 295-310.

[7] E. A. Coutsias, N.D. Kazarinoff, "The approximate functional formula for the theta function and Diophantine Gauss sums", Trans. Amer. Math. Soc., 350:2 (1998), 615-641.

[8] M. V. Berry, J. Goldberg, "Renormalisation of curlicues", Nonlinearity, 1:1 (1988), $1-26$.

[9] J. Marklof, "Limit theorems for theta sums", Duke Math. J., 97:1 (1999), 127-153.

[10] J. Marklof, "Theta sums, Eisenstein series, and the semiclassical dynamics of a precessing spin", Emerging applications of number theory (Minneapolis, MN, 1996), IMA Vol. Math. Appl., 109, Springer, New York, 1999, 405-450.

[11] A. Fedotov, F. Klopp, "Renormalization of exponential sums and matrix cocycles", Séminaire: Équations aux Dérivées Partielles. 2004-2005, Exp. XVI, École Polytech., Palaiseau, 2005, 12 pp. 
[12] Я.Г. Синай, "О распределении первой положительной суммы для последовательности независимых случайных величин", Теория вероятн. и ее примен., 2:1 (1957), 126-135; англ. пер.: Ya. G. Sinai, "On the distribution of the first positive sum for a sequence of independent random variables", Theory Probab. Appl., 2:1 (1957), 122-129.

[13] Ya. G. Sinai, C. Ulcigrai, "Renewal-type limit theorem for the Gauss map and continued fractions", Ergodic Theory Dynam. Systems, 28:2 (2008), 643-655.

[14] F. Cellarosi, "Renewal-type limit theorem for continued fractions with even partial quotients", Ergodic Theory Dynam. Systems (to appear); arXiv: 0802.4459, 2008.

[15] F. Schweiger, "Continued fractions with odd and even partial quotients", Arbeitsber. Math. Inst. Univ. Salzburg, 1982, № 4, 59-70.

[16] F. Schweiger, "On the approximation by continued fractions with odd and even partial quotients", Arbeitsber. Math. Inst. Univ. Salzburg, 1984, № 1-2, 105-114.

[17] C. Kraaikamp, A. Lopes, "The theta group and the continued fraction expansion with even partial quotients", Geom. Dedicata, 59:3 (1996), 293-333.

[18] J. Aaronson, An introduction to infinite ergodic theory, Math. Surveys Monogr., 50, Amer. Math. Soc., Providence, RI, 1997.

[19] M. Campanino, S. Isola, "Infinite invariant measures for non-uniformly expanding transformations of $[0,1]$ : weak law of large numbers with anomalous scaling", Forum Math., 8:1 (1996), 71-92.

Я. Г. Синай (Ya. G. Sinai)

Поступила в редакцию

Институт теоретической физики им. Л. Д. Ландау РАН;

15.08 .2008

Принстонский университет, Принстон, США

E-mail: sinai@math.princeton.edu 